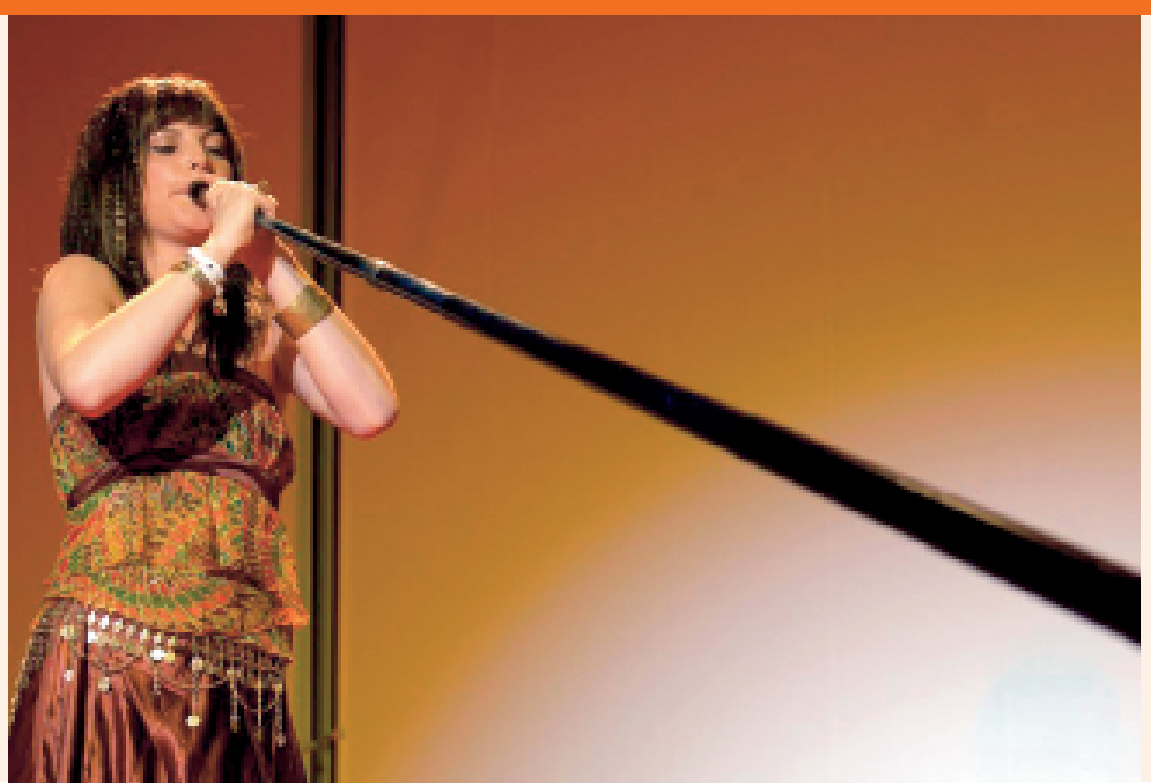

Die Rocklady Eliana Burki vibriert ins Rohr.

\title{
Das Alphorn für mehr Musik in der Medizin
}

\section{Bruno Fricker}

Korrespondenz:

SPECTRALAB Bruno Fricker Dipl. Physiker ETH

Brunnenmoosstrasse 7

CH-8802 Kilchberg

Tel. 0447155427

Fax 0447155447

www.spectralab.ch
Mächtige Alphornklänge verzaubern die Geräuschlandschaft des Zürcher Hauptbahnhofs: Die Rocklady Eliana Burki vibrierte ins Rohr. Unglaublich, welch mitreissende Klangmacht ein zierlicher Mund hervorzuzaubern vermag wenn er dieses Instrument beherrscht. «Musik als Motor» war das Thema eines ganz ungewöhnlichen Medizinsymposiums. Eliana behandelt mit Alphorn im Alpinen Kinderspital Davos Patienten mit zystischer Fibrose. Für die Kinder ist das mehr als nur Plausch, die Sekretdrainage ihrer kranken Lunge verbessert sich. Hier zeigt sich, wie Musik eine vegetative Heilwirkung anregen kann. Die Rocklady als Musiktherapeutin!

Die Schweizerische Fördergesellschaft für Psychofonie hält ihre 10. Jahrestagung ab. Einer der 100 weltbesten Neurowissenschaftler, Professor Lutz Jäncke von der Universität Zürich, erklärt in seinem mitreissenden Referat, wie sich das Gehirn unter Musikeinwirkung plastisch verändert, so dass, wer nachhaltig Musik hört oder noch besser - ausübt, ein neuer Mensch wird. Der Fortschritt zeigt sich durch heute messbare Volumenvergrösserung und vergrösserte Furchen in der gebuchteten Hirnrinde. Jede Funktion, die trainiert wird, kann gefördert werden, jede Funktion, die zuwenig benützt wird, verkümmert. Jede! Sogar die Muttersprache. Diese aus dem Krafttraining für Muskelzellen bestens bekannte Tatsache gilt auch für die Nervenzellen im Menschenhirn, von der Kindheit bis ins Greisenalter. Milliarden von Nervenverbindungen spriessen aus und werden effizienter, wenn sie gebraucht werden. Jäncke ist der Forscher, der diesen Vorgang exakt nachgemessen hat, womit Neuroplastizität nun als gesichertes Allgemeingut in die Neuropsychologie Eingang finden konnte. Leider hat die Medizin dieses Heilpotential noch nicht vollumfänglich entdeckt. Sonst würde sie der Musiktherapie Tür und Tor öffnen, die noch allzuoft ein nur kümmerliches Dasein fristet, in abgeschiedenen Nebenräumen der Spitäler. Von der musikalisch angeregten Neuroplastizität zur Rehabilitation in einem den ganzen Menschen umfassenden Sinn ist es aber nur ein kleiner logischer Schritt, der sich in der Medizin als grosser Paradigmenwechsel ankündigt.

«Mehr Musik in der Medizin» war deshalb der Slogan dieser öffentlichen Veranstaltung, die vom Jugendlichen bis zum längst pensionierten Hörer gut besucht war. Das Restaurant Au Premier öffnete dazu gleich zwei Säle. Jäncke erzählte aus seinen weltweit beachteten Forschungsarbeiten, die auf Kernspintomographie und fortgeschrittener EEG-Technik (Kohärenzanalyse und LORETA-Bildgebung eines Schweizer Physikers) basieren. Seine Publikationen sind bahnbrechend, sie sind in erstklassigen Fachzeitschriften erschienen und werden überall zitiert [1]. Im Kern hat er bisher gefunden, dass unser Kopf auf unablässiges Training angewiesen ist. Besonders gut eignet sich dafür die Musik, da sie die verschiedenen Areale gemeinsam aktiviert und den Musikern eine integrierte Topleistung abfordert und dadurch deren Gehirn fördert. Musiker sind deshalb in vielerlei Hinsicht dem Nichtmusiker voraus. Musik kann wie kein an- 
deres Training das jugendliche Hirn aufbauen, zum Guten organisieren und das alte Hirn vor dem Zerfall schützen.

Dass die Psychofonie-Gesellschaft Professor Jäncke sprechen liess, kommt nicht von ungefähr. Ist doch die Psychofonie selbst eine ganz besonders wirksame Musiktherapie, die obendrein sehr einfach und angenehm ist. Wer sich mit Psychofonie einen klaren Kopf verschafft, bleibt dabei, hört seine abgestimmte CD jahrelang. Weitere erschöpfende Information gibt die Homepage www.psychofonie.ch. Dort ist auch eine Liste von Therapiestellen für Psychofonie, die landauf, landab von etablierten Ärzten und Therapeutinnen gut zugänglich und kostengünstig angeboten wird. Gute Idee: Bei nicht kulanten Krankenkassen steht ein unkomplizierter therapieeigener Spendenfonds zur Verfügung.

\section{Literatur}

1 Munte TF, Altenmuller E, Jancke L. The musician's brain as a model of neuroplasticity. Nat Rev Neurosci 2002;3(6):473-8.

\section{Ein Lifting für Mona Lisa}

\section{Erhard Taverna}

Vielleicht hat sich der Meister selbst verliebt, denn das Bild nahm er mit in seinen Alterssitz nach Amboise an der Loire. Das Porträt der dritten Ehefrau des Kaufmanns Francesco del Giocondo hat seit Leonardo da Vinci zahlreiche Künstler inspiriert. So auch Urs Victor Burki, Gründer und Leiter der eigenen Klinik in Genf und ehemaliger Präsident der Schweizerischen Gesellschaft für Ästhetische Medizin (SSME).

Das Umschlagbild seines Buches «Kunst \& Ästhetische Chirurgie» zeigt die lächelnde Gioconda vor und nach der Operation. Eine provokative Konfrontation der damals erst 24jährigen Frau mit ihrem Ebenbild aus einer anderen Epoche. Sie sei ihm im Traum erschienen, erzählt Urs Burki, und er habe sie in einem barocken Konzertsaal unter Orgelbegleitung operiert. Das daraus folgende Gedankenexperiment ist eine faszinierende Einführung in die Arbeitsweise eines Künstlers, der das berühmte Modell mit dem Skalpell «nachbessert». Aufgrund eigener Proportionsanalysen werden die Gesichtspartien umgebaut: Augenunterlidplastik mit kleiner Muskelresektion, Osteotomie der Nase mit Exzision eines Knorpelstückes, Anhebung und Vergrösserung der Lippen, Modellierung der Wangen und des Kinns durch Filling und Mikroliposkulptur.

Das reichillustrierte Buch wendet sich an Fachkreise und ein breites Publikum. Es schildert eine Chirurgie der Gesichtsverjüngung, die Ästhetik mit persönlichen Merkmalen natürlich und harmonisch verbinden will. Der Autor begibt sich auf die Suche nach der Schönheit und belegt an zahlreichen historischen Beispielen die erstaunliche Konstanz dessen, was uns an einem Gesicht gefällt. Kronzeugen finden sich in unserer Kultur lückenlos, von der altägyptisch-griechisch-römischen Antike über die Renaissance bis zu Marilyn Monroe. Den Beispielen philosophischer Kommentare zum Schönheitsbegriff, ab den Pythagoreern bis zu Adornos ästhetischer Theorie, folgen die Proportionsstudien der Zeichner und Bildhauer. Gibt es eine Geometrie der Schönheit, lässt sich das Ideal vermessen? Leonardo da Vinci, Albrecht Dürer und viele andere entwickelten die Koordinaten des menschlichen Antlitzes. Gestützt auf ihre Studien, hat Urs Burki ein eigenes Schema erarbeitet, das er mit anschaulichen Skizzen und Fotos verständlich erklärt. Seine Gedanken zum Goldenen Schnitt, zur Fibonacci-Zahlenserie und den Tükken der Gesichtssymmetrie und Beobachtungsdistanz sind eine spannende Vorlesung über die minutiöse Planung eines ästhetisch-chirurgischen Eingriffes. Einer kurzen Würdigung der Pioniere seines Faches folgt das Hauptkapitel «Ästhetische Gesichtschirurgie». Gemäss Operateur entscheiden sich die meisten seiner Patienten zwischen vierzig und fünfundfünfzig Jahren für eine Veränderung. Konzise Darstellungen erläutern die häufigsten Eingriffe: Augenlidkorrekturen, Stirn- und Augenbrauenlifting, Gesichtsund Halslifting, Minilifting, Mikroliposuktion/ -skulptur, Oberflächenbehandlungen chemisch, abrasiv und mit Laser, Botulinumtoxin, Auffüllungen mit Hyaluronsäure oder Eigenfett, Rhinoplastik, Gesichtsknochenchirurgie, Oto- 


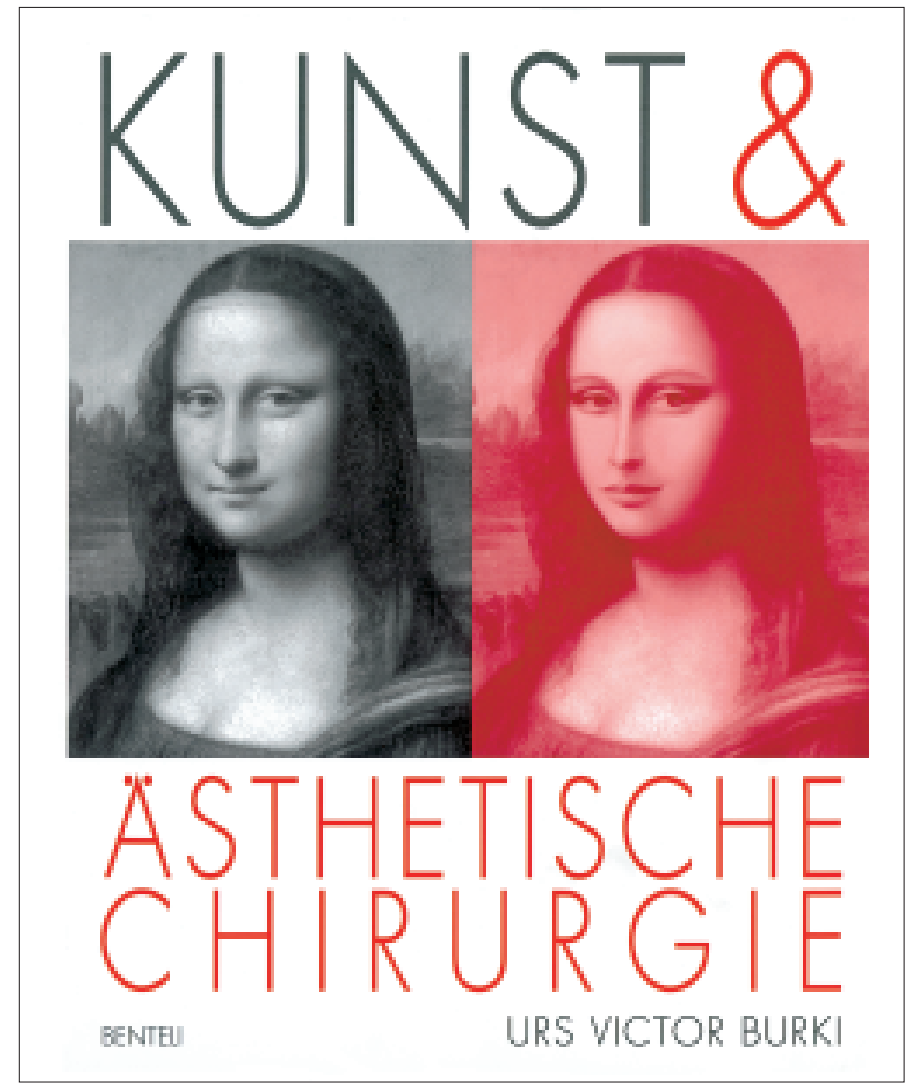

plastik und Lippenkorrekturen. Die wichtigsten Aussagen sind nach jedem Abschnitt zusammengefasst und ein postoperativer Kalender zeigt für jede Intervention den zeitlichen Ablauf bis zur Gesellschaftsfähigkeit. Eine Porträtgalerie als Werkausstellung dokumentiert den Weg zum optimalen Ergebnis, den einige wenige Erlebnisberichte abrunden.

Mona Lisa ist das Maskottchen der plastischen Chirurgengilde, denn schon im Buch der Psalmen steht: «Des Menschen Pracht ist ohne Bestand.» Es ist äusserst schwierig, nach der Buchlektüre selbstkritisch in einen Spiegel zu schauen. Doch das spricht für die verführerische Qualität eines hervorragenden und sehr ansprechend gestalteten Lehrbuches für Nichtspezialisten und Patienten und alle, die an dem komplexen Fachgebiet interessiert sind.

- Urs Victor Burki. Kunst \& Ästhetische Chirurgie. Bern: Benteli; 2005. 136 Seiten. 